\title{
The breastfeeding self-efficacy scale-short form: Psychometric characteristics in Portuguese pregnant women
}

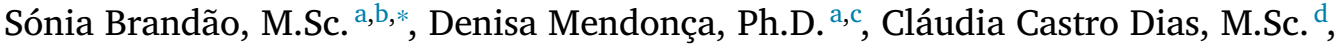 \\ Tiago Miguel Pinto, M.Sc. ${ }^{\mathrm{d}}$, Cindy-Lee Dennis, Ph.D. ${ }^{\text {eff }}$, Bárbara Figueiredo, Ph.D. ${ }^{\mathrm{d}}$ \\ a Instituto de Ciências Biomédicas Abel Salazar (ICBAS), University of Porto, Rua Jorge de Viterbo Ferreira 228, 4050-313 Porto, Portugal \\ ${ }^{\mathrm{b}}$ Centro Materno Infantil do Norte - Centro Hospitalar do Porto, Largo da Maternidade de Júlio Dinis, 4050-651 Porto, Portugal \\ ${ }^{\mathrm{c}}$ EPIUnit, Instituto de Saúde Pública, Universidade do Porto, Rua das Taipas 135, 4050-600 Porto, Portugal \\ ${ }^{\mathrm{d}}$ School of Psychology, University of Minho, Escola de Psicologia da Universidade do Minho, Campus de Gualtar 4710-057 Braga, Portugal \\ ${ }^{\mathrm{e}}$ Lawrence S. Bloomberg Faculty of Nursing University of Toronto, Suite 130, 155 College St, Toronto, ON M5T 1P8, Canadá \\ ${ }^{\mathrm{f}}$ Li Ka Shing Knowledge Institute St. Michael's Hospital, 209 Victoria St, Toronto, ON M5B 1T8, Canadá
}

\section{A R T I C L E I N F O}

\section{Keywords:}

Breastfeeding

Self-efficacy

Psychometric assessment

Pregnant women

Portuguese

\begin{abstract}
A B S T R A C T
Background: The Breastfeeding Self-Efficacy Scale-Short Form is a reliable instrument to measure mother's confidence in her ability to breastfeed. The Breastfeeding Self-Efficacy Scale-Short Form has traditionally been used postnatally, but evidence suggests that it can be used antenatally to identify mothers at-risk of requiring additional support to improve breastfeeding outcomes.

Objective: The aim of this study was to examine the psychometric characteristics of an antenatal version of the Breastfeeding Self-Efficacy Scale-Short Form, among pregnant Portuguese women.

Design: Methodological prospective study to examine the psychometric characteristics of the antenatal Portuguese version of the Breastfeeding Self-Efficacy Scale-Short Form.

Settings: Two public hospital units in the Northern Portugal.

Participants: The sample was comprised of 373 pregnant women recruited at 30-34 gestational weeks.

Methods: The original English version of the antenatal Breastfeeding Self-Efficacy Scale-Short Form was translated into Portuguese and the Portuguese antenatal version of the scale was tested in a sample of 373 pregnant women. To examine the psychometric characteristics of the Portuguese antenatal version of the scale, the maternal demographic variables and the depressive and anxiety symptomatology of the participants were examined. The predictive validity of the Portuguese antenatal version of the scale was studied according to infant feeding method at one, three, and six months postpartum.

Results: The Cronbach's alpha coefficient was 0.92. The antenatal version of the Breastfeeding Self-Efficacy ScaleShort Form scores were found to be associated with women's parity, educational level, occupational status, time they intend to breastfeed, and previous breastfeeding experience. Also, the antenatal version of the Breastfeeding Self-Efficacy Scale-Short Form scores significantly predicted exclusive breastfeeding at 1 month postpartum.

Conclusions: Results provided evidence that the antenatal Portuguese version of the Breastfeeding Self-Efficacy Scale-Short Form is a valid and reliable measure to assess breastfeeding self-efficacy in pregnant women. The antenatal version of the Breastfeeding Self-Efficacy Scale-Short Form could be a useful tool to assist Portuguese health professionals during routine prenatal care appointments to screen women with lower antenatal breastfeeding self-efficacy and, consequently, those that could be at risk for not initiating or early breastfeeding cessation.
\end{abstract}

\section{Introduction}

Breastfeeding is consistently associated with both maternal and child well being. The World Health Organization (WHO, 2003), the European
Commission for Public Health (Cattaneo et al., 2005; Cattaneoet al., 2010) and the American Academy of Pediatrics (Gartner et al., 2005) all recommend exclusive breastfeeding for the first six months of life, however despite high rates of breastfeeding initiation, exclusivity rates at six months postpartum remain low and a long-standing problem.

\footnotetext{
* Corresponding author.

E-mail addresses: soniabrandao.obstetricia@chporto.min-saude.pt (S. Brandão), dvmendon@icbas.up.pt (D. Mendonça), cdias@psi.uminho.pt (C.C. Dias), tmpinto@psi.uminho.pt (T.M. Pinto), cindylee.dennis@psi.utoronto.ca (C.-L. Dennis), bbfi@psi.uminho.pt (B. Figueiredo).
} 
In Portugal, the Breastfeeding Registration Report of the DirectorateGeneral of Health (DGS) reports that $98.6 \%$ of women initiate breastfeeding but only $76.7 \%$ are exclusively breastfeeding at hospital discharge. Further, at three months postpartum only $52.8 \%$ of women are still exclusively breastfeeding, rapidly decreasing to only $22.1 \%$ at six months (DGS, 2013). To promote exclusive breastfeeding until six months postpartum is currently a priority in Portugal. The UNICEF's "Baby Friendly Hospital" is ongoing in 14 public hospitals, and in some of them for over the last 10 years. Additionally, the DGS provides a web-site with information on breastfeeding, namely open publications and a list of public hospitals and local health centers with a so-called "breastfeeding corner". Breastfeeding corners provide daily individual support to overcome breastfeeding challenges and difficulties. Despite these programmes, rates are still low and more active initiatives are needed.

To improve exclusive breastfeeding rates, the early identification of women at risk of early breastfeeding cessation is important. Research has demonstrated that several socio-demographic factors, namely maternal age, educational level, marital status, and socio-economic status are associated with breastfeeding duration and exclusivity rates (Bonet et al., 2013; Haroon et al., 2013; Leahy-Warren et al., 2014; Onah et al., 2014).

However, most of these reasons to early breastfeeding cessation are non-modifiable variables that cannot be altered through health care interventions. For this reason, health professionals should target modifiable variables associated with positive breastfeeding outcomes. Breastfeeding self-efficacy is considered an important modifiable variable positively associated with breastfeeding duration and exclusivity (Dennis, 1999; Dennis \& Faux, 1999; Laanterä et al., 2012; Otsuka et al., 2014).

Breastfeeding self-efficacy theory (Dennis, 1999) consists in an application of Bandura's (1997) social cognitive theory and refers to a mother's confidence in her ability to breastfeed her new baby. It significantly influences (1) maternal intention to breastfeed, (2) how much effort she will put into breastfeeding, (3) whether she will have selfenhancing or self-defeating thought patterns, and (4) the emotional ability to respond to breastfeeding difficulties (Dennis, 1999; Dennis \& Faux, 1999; Dennis, 2003). Breastfeeding self-efficacy is influenced by several factors, namely (1) previous breastfeeding experience, (2) contact with mothers who have previously breastfed, (3) encouragement and support from others, such as family and health professionals, and (4) maternal physical and psychological state, such as fatigue, depression and anxiety (Dennis, 2006; Meedya et al., 2010; de Jager, Skouteris, Broadbent, Amir, \& Mellor, 2013; de Jager, Broadbent, Fuller-Tyszkiewicz, \& Skouteris, 2014).

The Breastfeeding Self-Efficacy Scale (BSES) was developed to measure breastfeeding self-efficacy (Dennis \& Faux, 1999). The BSES was originally designed as a 33-item instrument but following a second methodological study, several items were deleted using explicit reduction criteria and the BSES was shortened to 14 items (Dennis, 2003). Based on the encouraging reliability analysis of the new 14-item Breastfeeding Self-Efficacy Scale-Short Form (BSES-SF), construct validity was assessed using factor analysis, comparison of contrasted groups, and correlations with measures of similar constructs. Support for predictive validity was demonstrated through significant mean differences in infant feeding method at four and eight weeks postpartum. Demographic response patterns suggested that the BSES-SF is a unique tool to identify mothers at risk of prematurely discontinuing breastfeeding (Dennis, 2003; Dennis et al., 2011; Wheeler \& Dennis, 2013).

The BSES - SF has been translated into many different languages (Wutke \& Dennis, 2007; Tokat et al., 2010; Zubaran et al., 2010; Ip et al., 2012; Oliver-Roig et al., 2012; Bosnjak et al., 2012; Gerhardsson et al., 2014; Ip et al., 2016) and psychometrically tested among diverse samples (Creedy et al., 2003; Torres et al., 2003; Gregory et al., 2008; McCarter-Spaulding \& Dennis, 2010; Dennis et al., 2011; Dodt et al., 2012; Wheeler \& Dennis, 2013). These studies provided evidence that the BSES-SF is a reliable and valid measure to assess mother's confi- dence in her ability to breastfeed. The BSES-SF has traditionally been used postnatally with new mothers. However, evidence suggests that it can be used during pregnancy to identify mothers at-risk of early exclusive breastfeeding cessation, requiring additional breastfeeding support to improve breastfeeding outcomes. Studies carried out during the third trimester of pregnancy have shown that higher scores on the antenatal version of the BSES-SF significantly predicted breastfeeding initiation and duration (Creedy et al., 2003; Tokat et al., 2010; Dennis et al., 2011).

It should be referred that only the postnatal version of the BSES-SF has been previously translated into Portuguese from Brazil and validated to Brazilian postpartum women (Zubaran et al., 2010). Moreover, it is necessary to emphasize that the Portuguese from Brazil, although sharing the same roots with the original Portuguese spoken in Portugal, is idiomatically different from the latter. The wide cultural differences between the two countries would imply the validation of the scale in both countries.

The antenatal version of BSES-SF could be a useful tool to assist Portuguese health professionals during routine prenatal care appointments to screen women with lower antenatal breastfeeding self-efficacy and, consequently, those that could be at risk for not initiating or early breastfeeding cessation.

The BSES-SF application during pregnancy can also be a powerful tool to design and implement new interventions on breastfeeding promotion. In Portugal, the childbirth preparation courses carried out by the National Health Service, starting between the 28th and the 30th week of pregnancy (DGS, 2016), are a privileged moment for the healthcare professionals to discuss with the parents-to-be the feeding method to be provided to the child. In these classes, it is also essential to understand the parents' -to-be feelings about breastfeeding, sharing with them knowledge about its importance, and demystifying its myths. By doing so, healthcare professionals aim to encourage the mothers to initiate and continue exclusive breastfeeding (WHO, 2003; Cattaneoet al. 2005; Gartner et al., 2005; Cattaneoet al., 2010).

The aim of this study was to examine the psychometric characteristics of an antenatal version of the Breastfeeding Self-Efficacy Scale-Short Form among pregnant Portuguese women.

\section{Methods}

This study is part of a larger longitudinal study examining the association between breastfeeding and postpartum depression conducted in Northern Portugal from October 2012 to May 2014. The two public hospital units that participate in the study are UNICEF's "Baby Friendly Hospital" certified.

The study was designed to examine the psychometric characteristics of an antenatal version of the Breastfeeding Self-Efficacy Scale-Short Form among pregnant Portuguese women. Participants were assessed at the third trimester of pregnancy (between 30 and 34 gestational weeks), and then at the first, third and sixth months postpartum

\section{Participants}

The sample is comprised of 373 pregnant women. Pregnant women were eligible to participate in the study if they were between 30 and 34 gestational weeks, Portuguese speaking, and over the age of 17 years. Women were excluded if they had multiple fetuses or any health issues that could interfere with breastfeeding (e.g., previous breast surgery).

\section{Instruments}

Breastfeeding Self-Efficacy Scale - Short Form (BSES-SF). The BSES-SF is a 14-item self-report instrument (Dennis \& Faux, 1999; Dennis, 2003) where all items are presented positively and scored using a five-point Likert-type scale, ranging from one (not at all confident) to five (always 
confident). Total scores of the scale can range from 14 to 70 . Higher scores indicate higher levels of breastfeeding self-efficacy. The psychometric characteristics of the BSES-SF antenatal version were previously examined in two other studies and the item stem was changed from "I can" to "I think I can..." at the beginning of each item. The Cronbach's alpha coefficient was respectively 0.87 and 0.84 for the antenatal assessment of the BSES-SF (Tokat et al., 2010; Dennis et al., 2011).

The BSES-SF used in this study was the antenatal form that aims to evaluate breastfeeding confidence during pregnancy, before women actually experienced breastfeeding their child.

State Anxiety Inventory. The state anxiety subscale (STAI-S) of StateTrait Anxiety Inventory (Spielberger, 1985) was used to assess anxiety symptomatology during pregnancy. The STAI-S is a self-report measure that consists of 20 items scored on a 4-point Likert-type scale where higher scores indicate higher levels of anxiety symptomatology. Several studies have used this instrument with women during pregnancy and the postpartum period. The Portuguese version of STAI-S has shown good internal consistency (Cronbach alpha $=0.87$ ) with a score equal or higher than 40 recommended to identify high-anxiety states in pregnant women (Tendais et al., 2014).

Edinburgh Postnatal Depression Scale. The Edinburgh Postnatal Depression Scale (EPDS; Cox et al., 1987) was used to assess depressive symptomatology during pregnancy. The EPDS is a 10-item self-report scale scored on a 4-point Likert-type scale where higher scores indicate higher levels of depressive symptomatology. Several studies have used this instrument with women during pregnancy and the postpartum period, namely in Portugal (e.g., Figueiredo et al., 2014). The Portuguese version of the EPDS has shown good internal consistency (Cronbach Alpha $=0.85-0.82$ ) and a score equal or higher than 9 is recommended to screen for depression in pregnant women (Tendais et al., 2014).

\section{Translation procedures}

The translation procedure was made by two independent bilingual translators who translated the BSES-SF into Portuguese and backtranslated it to English. To ensure content, semantic, and technical equivalence, the translation was performed using the standard procedure of translation and blind back-translation. The two translators discussed the backward translation and consensus was reached through discussion. To assess content appropriateness, three breastfeeding expert midwives reviewed the translated BSES-SF to ensure accuracy, clarity, and suitability of the items for Portuguese culture. Finally, a pilot study was conducted with 15 pregnant women in order to test the comprehensibility and legibility of the translated BSES-SF.

Following the university and hospitals ethics committee approvals, participants were recruited by trained research assistants in two public outpatient hospital units in the Northern Portugal.

\section{Data collection}

Eligible and consenting women were recruited into the study and administered the baseline questionnaire that included sociodemographic questions, the BSES-SF, and measures of anxiety and depression symptoms. Follow-up questionnaires to evaluate infant feeding method were sent by email to all the participants at one, three, and six months postpartum. For this study, infant feeding method breastfeeding was defined according the following categories: (a) exclusive breastfeeding (i.e., breast milk only), (b) partial breastfeeding (i.e., breast milk and formula), and (c) bottle-feeding (i.e., no breast milk at all). Those who did not return their questionnaires within one week of mailing received a reminder telephone call. From the 525 women approached, 447 (85.1\%) were eligible, and $386(86.4 \%)$ agreed to participate and signed the informed consent. After delivery, 13 (3.4\%) women were excluded because they had a premature birth.
From the total sample of 373 pregnant women, 293 completed data on infant feeding method at one-month follow-up moment, 274 at threemonth follow-up moment, and 241 at six-month follow-up moment.

\section{Data analysis}

The internal consistency of the antenatal version of the translated BSES-SF was assessed using the following criteria: (1) Cronbach's alpha coefficient, (2) corrected item-total correlation, and (3) alpha estimate when an item was dropped from the scale. Poorly functioning items were defined as: (1) items that when deleted increased the coefficient alpha by more than 0.10 or (2) items that had a corrected item-total correlation less than 0.30 . This criterion was considered according with previous recommendations (Field, 2005) and based on the same criterion used in previous BSES psychometric investigations (Dennis, 2003; Creedy et al., 2003; Tokat et al., 2010; Zubaran et al., 2010). An exploratory factor analysis (principal axis factoring analysis with orthogonal rotation-varimax) was performed to test the construct validity of the instrument and the appropriateness of the scale. All items were examined across both factors to assess loading complexity employing a 0.40 loading criterion. This criterion was considered according with previous recommendations (Field, 2005) and based on the same criterion used in previous BSES psychometric investigations (e.g. Creedy et al., 2003; Dennis, 2003; Dennis et al., 2010).

To examine the antenatal version of the BSES-SF criteria validity differences on the breastfeeding self-efficacy were analyzed according with maternal demographic variables, such as maternal age groups, marital status, occupational status, and educational level, and breastfeeding selfefficacy using independent samples $t$-tests and one-way analyses of variance (ANOVA). To further examine the antenatal version of the BSESSF criteria validity, differences on breastfeeding self-efficacy according with depressive and anxiety symptomatology were assessed using independent samples $t$-tests. To assure the construct validity of the scale, it was hypothesized that pregnant multiparous women with previous experience of breastfeeding would have higher levels of breastfeeding selfefficacy than primiparous with no previous breastfeeding experience. It was also hypothesized that pregnant women who intended to breastfeed for a long period would have higher levels of breastfeeding self-efficacy. Independent samples $t$-tests were used to assess these hypotheses. Predictive validity was studied analysing the translated antenatal version of the BSES-SF mean scores in terms of infant feeding method at one, three and six months postpartum. We assessed the hypotheses using independent samples $t$-tests and one-way ANOVA. The statistical significance level was set at 0.05 . The statistical analyses were performed using SPSS for Windows version 22.

\section{Results}

\section{Description of sample}

The mean age of participants was 30 years $(S D=5.03)$, ranging from 18 to 44 years. The majority were primiparous (79.6\%), married or cohabitating $(77.4 \%)$, and currently employed $(70.1 \%)$. Over half of the participants had more than 12 years of education (53.4\%) and all intended to initiate breastfeeding with $69.8 \%$ reporting they planned to breastfeed for more than six months. Regarding multiparous women, $39.2 \%$ had breastfed their previous infant for less than six months. Overall, $34.1 \%(n=127)$ of participants presented with high-anxiety symptoms (STAI-S $\geq 40)$, and $40.8 \%(n=152)$ had depressive symptoms (EPDS $\geq$ 9; see Table 1).

Missing values were identified in some of the study variables (e.g., age, marital status, occupational status, years of schooling, anxiety symptoms, time that intent breastfeed and time of breastfeeding previous babies). However, these missing values were as missing completely at random (MAR) and their percentages ranged between $0.3 \%$ (anxiety symptoms) and 6.7\% (time that intent breastfeed). 
Table 1

Characteristics of the study sample.

\begin{tabular}{llll}
\hline & & $\mathrm{N}=373$ & $\%$ \\
\cline { 2 - 4 } & & $\mathrm{n}$ & \\
\hline Sociodemographic characteristics & & & 14.4 \\
Age & $<25$ & 52 & 65.2 \\
& $25-35$ & 236 & 20.4 \\
Marital status & $>35$ & 74 & 77.4 \\
Occupational status & Married/Cohabiting & 287 & 22.7 \\
& Single/Divorced/Widow & 84 & 70.1 \\
Years of schooling & Employed & 260 & 29.9 \\
Parity & Unemployed/Household/student & 111 & 46.6 \\
Psychological Characteristics & $\leq 12$ & 173 & 53.4 \\
Anxiety Symptoms & $>12$ & 198 & 79.6 \\
Depression symptoms & Primiparous & 297 & 20.4 \\
Intention for time of breastfeeding & STAI-S $<40$ & 76 & 65.9 \\
Time that intent breastfeed & STAI-S $\geq 40$ & & 34.1 \\
Breastfeeding experience in & EPDS $<9$ & 245 & 59.2 \\
multiparous & EPDS $\geq 9$ & 127 & 40.8 \\
Time of breastfeeding previous babies & $\leq 6$ months & 221 & 32 \\
& $>6$ months & 152 & 30.2 \\
\hline
\end{tabular}

Note. Missing values were considered as missing completely at random

Table 2

BSES-SF items with principal components factor loadings and reliability results.

\begin{tabular}{|c|c|c|c|}
\hline & Loading & Corrected item-total correlation & Cronbach's alpha if item deleted \\
\hline \multicolumn{4}{|l|}{ BSES-SF factor 1} \\
\hline 3. I think I can always breastfeed my baby without using formula as a supplement & 0.55 & 0.66 & 0.90 \\
\hline 4. I think I can always ensure that my baby is properly latched on for the whole feeding & 0.60 & 0.74 & 0.90 \\
\hline 5. I think I can always manage the breastfeeding situation to my satisfaction & 0.75 & 0.77 & 0.89 \\
\hline 7. I think I can always keep wanting to breastfeed & 0.85 & 0.76 & 0.89 \\
\hline 8. I think I can always comfortably breastfeed with my family members present & 0.66 & 0.57 & 0.92 \\
\hline 9. I think I can always be satisfied with my breastfeeding experience & 0.81 & 0.77 & 0.89 \\
\hline 10. I think I can always deal with the fact that breastfeeding can be time consuming & 0.75 & 0.73 & 0.90 \\
\hline 12. I think I can always continue to breastfeed my baby for every feeding & 0.63 & 0.74 & 0.90 \\
\hline \multicolumn{4}{|l|}{ BSES-SF factor 2} \\
\hline 1. I think I can always determine that my baby is getting enough milk & 0.82 & 0.54 & 0.78 \\
\hline 2. I think I can always successfully cope with breastfeeding like I have with other challenging tasks & 0.52 & 0.65 & 0.90 \\
\hline 6. I think I can always manage to breastfeed even if my baby is crying & 0.60 & 0.59 & 0.77 \\
\hline 11. I think I can always finish feeding my baby on one breast before switching to the other breast & 0.61 & 0.59 & 0.76 \\
\hline 13. I think I can always manage to keep up with my baby's breastfeeding demands & 0.60 & 0.55 & 0.77 \\
\hline 14. I think I can always tell when my baby is finished breastfeeding & 0.72 & 0.68 & 0.73 \\
\hline
\end{tabular}

Notes. $\alpha=$ Cronbach's alpha.

\section{Internal consistency}

The Cronbach's alpha coefficient for the translated antenatal version of the BSES-SF was 0.92 and was not increased by more than 0.1 if any of the items were deleted. All corrected item-total correlations were positive and above 0.30 . The mean score for the antenatal Portuguese version of BSES - SF in this sample was $57.93(S D=7.90)$, ranging from 25 to 70 (see Table 2).

\section{Construct validity}

Factor analysis. The Kaiser-Meyer-Olkin measure of sampling adequacy was good $(\mathrm{KMO}=0.94)$. The principal component analysis yielded a two-factor solution with eigenvalues greater than one that explained $60.8 \%$ of the variance. Factor 1 had an eigenvalue of 7.45 , which explained $53.2 \%$ of the variance and comprised eight items with loadings higher than 0.40 (ranging from 0.55 to 0.85 ). Item examination revealed that Factor 1 assesses the thoughts, perceptions, and beliefs of pregnant women about their efficacy to breastfeed. Factor 2 had an eigenvalue of 1.06 , which explained $7.6 \%$ of the variance and was comprised of six items with loadings higher than 0.40 (ranging from 0.52 to 0.82 ). Item examination indicated that Factor 2 assesses specific tasks that pregnant women recognized that they have to accomplish for successful breastfeeding (see Table 2). However, the placement of the "elbow" in the figure related Cattell's scree test (Cattell, 1966) indicated retaining only one factor (see Fig. 1).

\section{Criterion validity}

Concurrent validity. No significant differences were found on the BSES - SF scores according to maternal age groups and marital status. Significant differences on the BSES-SF scores were found according with parity, $t(371)=-2.60, p<.05$. Primiparous women reported lower BSES-SF scores $(M=57.51, S D=8.02)$ than multiparous $(M=59.59, S D=7.25)$. 
Scree Plot

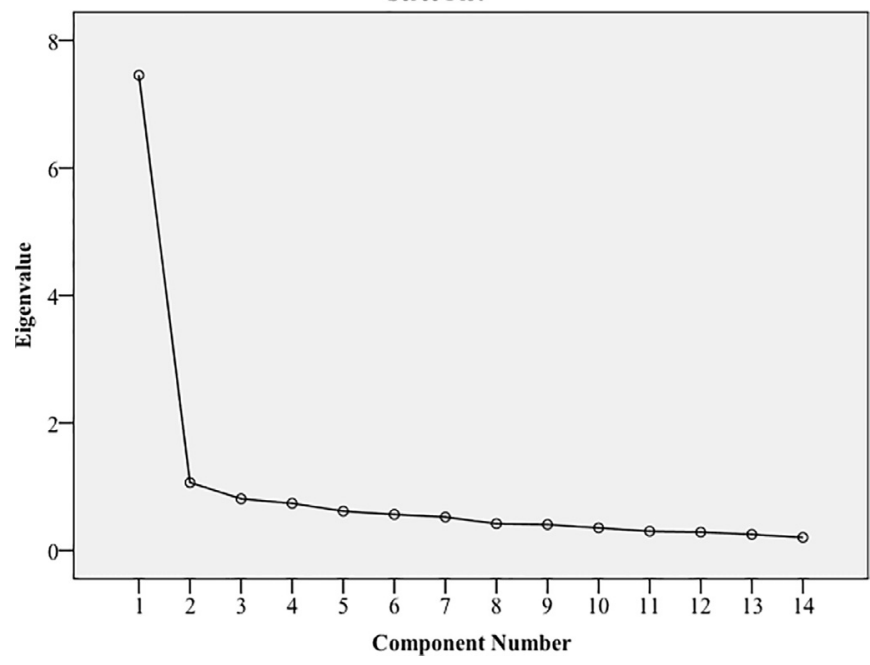

Fig. 1 Scree plot for factor analysis.

Also, significant differences were found on the BSES-SF scores according with education level, $t(369)=4.80 p<.01)$. Pregnant women who have 12 years of schooling or less reported higher scores on the BSESSF $(M=59.98, S D=7.13)$ than those who have more than 12 years of schooling $(M=56.14, S D=8.16)$. Upon further examination, this difference was related to parity. Primiparous pregnant women who have more than 12 years of schooling reported lower scores on the BSESSF $(M=55.53, S D=8.20)$ than those who have less than or equal to 12 years of schooling $(M=59.71, S D=7.23), t(294)=4.63, p<.01$. In multiparous women, these differences were not significant, $t(73)=1.62$, $p=0.110$.

In addition, significant differences were found on the BSES-SF scores according to occupational status, $t(369)=-2.24 p<.05$. Pregnant women who were unemployed, household or students reported higher scores on the BSES-SF $(M=59.36, S D=7.78)$ than those who were currently employed $(M=57.37, S D=7.91)$. Upon further examination, these differences were related to parity. Primiparous pregnant women who were currently employed reported lower scores on the BSES-SF $(M=56.57, S D=7.97)$ than those who were unemployed, household or students $(M=59.69, S D=7.75), t(294)=-3.14, p<.05$. In multiparous women, these differences were not significant, $t(73)=1.31, p=0.194$.

Moreover, significant differences were found on the BSES-SF scores according to breastfeeding intention, $t(346)=-4.01, p<.01$. Pregnant women who intend to breastfeed for less than six months reported lower BSES-SF scores $(M=55.35, S D=8.13)$ than those who intend to breastfeed for more than six months $(M=58.97, S D=7.54)$. Significant differences in BSES-SF scores were also found to be related with previous breastfeeding experience, $t(72)=-2.96, p<.05$. Multiparous pregnant women who breastfed the previous infant for less than six months reported lower BSES-SF scores $(M=56.62, S D=7.00)$ than multiparous pregnant women who breastfed the previous infant for more than six months $(M=61.51, S D=6.89)$.

Significant differences were found in BSES-SF scores according to anxiety, $t(370)=4.80, p<.01$. Women with high-anxiety symptoms (STAI-S score higher or equal to 40) reported significantly lower BSES$S F$ scores $(M=55.82, S D=8.61)$ than those with low-anxiety symptoms $(M=59.05, S D=7.30)$. Similarly, significant differences were found in BSES-SF scores according to depressive symptomatology. Women with an EPDS score higher or equal to 9 reported significantly lower BSES-SF scores $(M=56.70, S D=8.19)$ than women with an EPDS score lower to $9(M=58.78, S D=7.60), t(371)=2.51, p<.05$.

Predictive validity. Significant differences were found on the BSESSF scores according to infant feeding method at the first month post- partum, $F(2,290)=4,61 p<.05$. Women who were exclusively breastfeeding at the first month postpartum reported higher scores on the BSES-SF $(M=58.38, S D=7.40)$ than those who were in mixed feeding $(M=56.11, S D=7.72)$ or had discontinued breastfeeding $(M=53.95$, $S D=9.49$ ). No differences were found in the BSES-SF scores according to infant feeding method or exclusivity at three and six months postpartum.

\section{Discussion}

The BSES-SF was developed to measure the mother's confidence in her ability to breastfeed their infant. In this study, the antenatal version of the BSES-SF showed good psychometric characteristics when administered during the third trimester of pregnancy. Evidence from the present study supports the Portuguese version of the BSES-SF as a valid and reliable instrument to measure breastfeeding self-efficacy in pregnant women. The Cronbach's alpha for the antenatal Portuguese version of the BSES - SF scale was high and is similar to those of other studies that examined the internal consistency of the BSES-SF during the third trimester of pregnancy (0.87-0.84; Tokat et al., 2010; Dennis et al., 2011). Specifically, the Cronbach's alpha of 0.92 is similar to the 0.94 reported in the original BSES-SF study (Dennis, 2003), which provides evidence for the international validity of the BSES - SF scale, while reflecting the results of the other international versions of the scale (Wutke \& Dennis, 2007; Gregory et al., 2008; Tokat et al., 2010; McCarter-Spaulding \& Dennis, 2010; Zubaran et al., 2010; Dodt et al., 2012; Bosnjak et al., 2012; Oliver-Roig et al., 2012; Ip et al., 2012; Gerhardsson et al., 2014). The overall mean score of the antenatal Portuguese version of the BSES-SF is similar, not only to the original BSESSF mean score (Dennis, 2003), but also to the scores obtained in previous studies with the antenatal version of the scale (Tokat et al., 2010; Dennis et al., 2011). These findings suggest that similar reliability for both the original and the translated versions of the BSES-SF.

The BSES-SF has always been presented as a unidimensional scale with no subscales (Wutke \& Dennis, 2007; Gregory et al., 2008; Tokat et al., 2010; McCarter-Spaulding \&Dennis, 2010; Zubaran et al., 2010; Dodt et al., 2012; Bosnjak et al., 2012; Oliver-Roig et al., 2012; Ip et al., 2012; Gerhardsson et al., 2014). However, this study found that the principal component analysis yielded a two-factor solution with eigenvalues greater than one, even with the Factor 2 of the scale only explaining $7.6 \%$ of the variance. Additionally, the Cattell's scree tests indicate that only one factor should be considered. Taking this into account, despite presenting the scale with the two factors in the Factor analysis, a unidimensional scale was maintained for the calculation of both concurrent validity and predictive validity, consistent with the referred previous works. Also, no clinical utility was considered in having two subscales with only 14 items. Furthermore, the two factors do not alter the way clinical care is provided. However, considering that there is no confirmation of other studies for the existence of one or two factors in the antenatal version of the scale, we consider appropriate to maintain the scale as unidimensional, warning for the possibility of the existence of two factors.

The antenatal Portuguese version of the BSES-SF showed good criteria validity. Demographic factors have consistently been related with breastfeeding duration (Dennis, 2002; Meedya et al., 2010; Onah et al., 2014). In this study, significant differences were found in breastfeeding self-efficacy as function of maternal education. Pregnant women with higher educational levels reported lower breastfeeding self-efficacy. While this result is not consistent with previous research suggesting that higher maternal education was positively associated with breastfeeding initiation, duration and exclusivity (Dennis, 2002; Van Rossem, 2009; Tokat et al., 2010), the present study only targeted pregnant women who reported their breastfeeding self-efficacy before they actually experienced breastfeeding. Moreover, in the present study, differences were only found in primiparous pregnant women. The differences in occupational status may be explained by the time that employed women 
intend to be at home with their babies, since maternal leave in Portugal is fully paid for four months or partially paid for five months. This contrasts with the six-month exclusive breastfeeding period. These facts combined pressure women to early cease breastfeeding or to compromise in strategies to be able to breastfeed after returning to work (Bai et al., 2015).

The hypothesis that the BSES-SF was related with other psychological constructs was supported and provides further evidence of good criteria validity of the BSES-SF. Women with higher anxiety and depressive symptomatology reported lower breastfeeding self-efficacy. This result is congruent with those of previous studies reporting that women with depression during pregnancy are at higher risk for early cessation of exclusive breastfeeding and shorter breastfeeding duration (Figueiredo et al., 2014; Dias \& Figueiredo, 2015).

Banduras' theory (1977) states that self-efficacy is an individual's conviction that he or she can successfully perform certain tasks or behaviours in a given situation. Dennis (1999) applied Bandura's selfefficacy theory to breastfeeding, considering that breastfeeding selfefficacy is influenced by four main sources of information: performance accomplishments (e.g., past breastfeeding experiences), vicarious experiences (e.g., watching other women breastfeed), verbal persuasion (e.g., encouragement from influential others), and physiological responses (e.g., fatigue, stress, anxiety and depression). Building upon the performance accomplishment source of self-efficacy and consistent with previous studies, multiparous women had significantly higher breastfeeding self-efficacy scores than those who were primiparous (Dennis, 2003; Creedy et al., 2003). In addition, women who intended to breastfeed for a longer period reported higher breastfeeding self-efficacy scores. Moreover, building upon the physiological responses source of self-efficacy, women with higher anxiety and depressive symptomatology reported lower breastfeeding self-efficacy. Building upon these clinical findings, significant differences were found on the antenatal BSES-SF scores as function of exclusive breastfeeding at one month postpartum. This result is consistent with previous research (Tokat et al., 2010; Dennis et al., 2011) and provides evidence for BSES-SF predictive validity.

The present study presents several limitations. Data were collected only in two hospitals and there was a lower proportion of multiparous than primiparous women. Despite these limitations, these findings suggest that the BSES-SF is a reliable and valid instrument to assess breastfeeding self-efficacy in Portuguese women.

\section{Conclusions}

These results provide implications for clinical practice and research. The BSES-SF could be a useful tool to assist Portuguese health professionals during routine prenatal care appointments to screen women with lower antenatal breastfeeding self-efficacy and, consequently, those that could be at risk for not initiating or early breastfeeding cessation. The BSES-SF could also be a useful tool to design new interventions on breastfeeding promotion during pregnancy.

\section{Acknowledgements}

We are grateful to everyone who participated throughout the research process, namely the ones who translate de original BSES-SF to Portuguese and the midwifes who reviewed the translated BSES-SF. We also thank all the women who participated in the study.

\section{Source of funding}

This research was supported by FEDER Funds through the Programa Operacional Factores de Competitividade-COMPETE and by National Funds through FCT - Fundação para a Ciência e a Tecnologia under the project: PTDC/SAU/SAP/116738/2010. This study was conducted at Psychology Research Centre (UID/PSI/01662/2013), University of Minho, and supported by the Portuguese Foundation for Sci- ence and Technology and the Portuguese Ministry of Education and Science through national funds and co-financed by FEDER through COMPETE2020 under the PT2020 Partnership Agreement (POCI-01-0145FEDER-007653).

\section{References}

Bai, D.L., Fong, D.Y.T., Tarrant, M., 2015. Factors associated with breastfeeding duration and exclusivity in mothers returning to paid employment postpartum. Maternal and Child Health Journal 19 (5), 990-999.

Bandura, A., 1977. Self-efficacy: toward a unifying theory of behavioral change. Psychological Review 84, 191-215.

Bonet, M., Marchand, L., Kaminski, M., Fohran, A., Betoko, A., Charles, M.A., Blondel, B., 2013. Breastfeeding duration, social and occupational characteristics of mothers in the French 'EDEN Mother-Child'Cohort. Maternal and Child Health Journal 17 (4), 714-722.

Bosnjak, A.P., Rumboldt, M., Stanojevic, M., Dennis, C.L., 2012. Psychometric Assessment of the Croatian Version of the Breastfeeding Self-Efficacy Scale-Short Form. Journal of Human Lactation 28 (4), 565-569.

Cattaneo, A., Burmaz, T., Arendt, M., Nilsson, I., Mikiel-Kostyra, K., Kondrate, I., Fallon, M., 2010. Protection, promotion and support of breast-feeding in Europe: progress from 2002 to 2007. Public Health Nutrition 13 (6), 751-759.

Cattaneo, A., Yngve, A., Koletzko, B., Guzman, L.R., 2005. Protection, promotion and support of breast-feeding in Europe: current situation. Public Health Nutrition 8 (1), 39-46.

Cattell, R.B., 1966. The scree test for the number of factors. Multivariate Behavioral Research 1 (2), 245-276.

Cox, J.L., Holden, J.M., Sagovsky, R., 1987. Detection of postnatal depression: development of the 10-item Edinburgh depression scale. British Journal of Psychiatry 150, 782-786.

Creedy, D.K., Dennis, C.L., Blyth, R., Moyle, W., Pratt, J., De Vries, S.M., 2003. Psychometric characteristics of the breastfeeding self-efficacy scale: Data from an Australian sample. Research in Nursing \& Health 26 (2), 143-152.

de Jager, E., Skouteris, H., Broadbent, J., Amir, L., Mellor, K., 2013. Psychosocial correlates of exclusive breastfeeding: a systematic review. Midwifery 29 (5), 506-518.

de Jager, E., Broadbent, J., Fuller-Tyszkiewicz, M., Skouteris, H., 2014. The role of psychosocial factors in exclusive breastfeeding to six months postpartum. Midwifery 30 (6), 657-666.

Dennis, C.L., 1999. Theoretical underpinings of breastfeeding confidence: A self-efficacy framework. Journal of Human Lactation 15 (3), 195-202.

Dennis, C.L., 2002. Breastfeeding initiation and duration: 1990-2000 literature review. Journal of Obstetric, Gynecologic, and Neonatal Nursing 31 (1), 12-32.

Dennis, C.L., 2003. The breastfeeding self-efficacy scale: Psychometric assessment of the short form. Journal of Obstetric, Gynecologic, \& Neonatal Nursing 32 (6), 734-744.

Dennis, C.L., 2006. Identifying predictors of breastfeeding self-efficacy in the immediate postpartum period. Research in Nursing \& Health 29 (4), 256-268.

Dennis, C.L., Faux, S., 1999. Development and psychometric testing of the Breastfeeding Self-Efficacy Scale. Research in Nursing and Health 22 (5), 399-409.

Dennis, C.L., Heaman, M., Mossman, M., 2011. Psychometric testing of the breastfeeding self-efficacy scale-short form among adolescents. Journal of Adolescent Health 49 (3), 265-271.

Dias, C.C., Figueiredo, B., 2015. Breastfeeding and depression: A systematic review of the literature. Journal of Affective Disorders 171, 142-154.

Direção Geral de Saúde. (2013). Registo do Aleitamento Materno - Relatório de Janeiro a Dezembro de 2013. Direção Geral de Saúde.

Direção Geral de Saúde. (2016). Programa Nacional para a vigilância de gravidez de baixo risco. Direção Geral de Saúde.

Dodt, R.C.M., Ximenes, L.B., Almeida, P.C., Oria, M.B., Oliveira, C.L.N., 2012. Psychometric and maternal sociodemographic assessment of the breastfeeding self-efficacy scale-short form in a brazilian sample. Journal of Nursing Education and Practice 2 (3), 66-73.

Field, A., 2005. Discovering Statistics using SPSS. SAGE Publications, London.

Figueiredo, B., Canário, C., Field, T., 2014. Breastfeeding is negatively affected by prenatal depression and reduces postpartum depression. Psychological Medicine 44 (5), 927-936.

Gartner, L.M., Morton, J., Lawrence, R.A., et al., 2005. Breastfeeding and the use of human milk. Pediatrics 115, 496-506.

Gerhardsson, E., Nyqvist, K.H., Mattsson, E., Volgsten, H., Hildingsson, I., Funkquist, E.L., 2014. The Swedish Version of the Breastfeeding Self-Efficacy Scale-Short Form Reliability and Validity Assessment. Journal of Human Lactation 30 (3), 340-345.

Gregory, A., Penrose, K., Morrison, C., Dennis, C.L., MacArthur, C., 2008. Psychometric properties of the Breastfeeding Self-Efficacy Scale-Short Form in an ethnically diverse UK sample. Public Health Nursing 25 (3), 278-284.

Haroon, S., Das, J.K., Salam, R.A., Imdad, A., Bhutta, Z.A., 2013. Breastfeeding promotion interventions and breastfeeding practices: a systematic review. BMC Public Health 13, S3-S20.

Ip, W.Y., Gao, L.L., Choi, K.C., Chau, J.P.C., Xiao, Y., 2016. The short form of the breastfeeding self-efficacy scale as a prognostic factor of exclusive breastfeeding among mandarin-speaking Chinese mothers. Journal of Human Lactation 32 (4), 711-720.

Ip, W.Y., Yeung, L.S., Choi, K.C., Chair, S.Y., Dennis, C.L., 2012. Translation and validation of the Hong Kong Chinese version of the breastfeeding self-efficacy scale-short form. Research in Nursing \& Health 35 (5), 450-459.

Laanterä, S., Pietilä, A.M., Ekström, A., Pölkki, T., 2012. Confidence in breastfeeding among pregnant women. Western Journal of Nursing Research 34 (7), 933-951. 
Leahy-Warren, P., Mulcahy, H., Phelan, A., Corcoran, P., 2014. Factors influencing initiation and duration of breast feeding in Ireland. Midwifery 30 (3), 345-352.

McCarter-Spaulding, D.E., Dennis, C.L., 2010. Psychometric testing of the breastfeeding self-efficacy scale-short form in a sample of black women in the United States. Research in Nursing \& Health 33 (2), 111-119.

Meedya, S., Fahy, K., Kable, A., 2010. Factors that positively influence breastfeeding duration to 6 months: a literature review. Women and Birth 23 (4), 135-145.

Oliver-Roig, A., d'Anglade-González, M.L., García-García, B., Silva-Tubio, J.R., RichartMartínez, M., Dennis, C.L., 2012. The Spanish version of the breastfeeding self-efficacy scale-short form: Reliability and validity assessment. International Journal of Nursing Studies 49 (2), 169-173.

Onah, S., Osuorah, D.I.C., Ebenebe, J., Ezechukwu, C., Ekwochi, U., Ndukwu, I., 2014. Infant feeding practices and maternal socio-demographic factors that influence practice of exclusive breastfeeding among mothers in Nnewi South-East Nigeria: a cross-sectional and analytical study. International Breastfeeding Journal 9 (1), $1-18$.

Otsuka, K., Taguri, M., Dennis, C.L., et al., 2014. Effectiveness of a breastfeeding self-efficacy intervention: do hospital practices make a difference? Maternal and Child Health Journal 18 (1), 296-306.

Spielberger, C.D., 1985. Assessment of state and trait anxiety: conceptual and methodological issues. Southern Psychologist 2, 6-16.
Tendais, I., Costa, R., Conde, A., Figueiredo, B., 2014. Screening for depression and anxiety disorders from pregnancy to postpartum with the EPDS and STAI. The Spanish Journal of Psychology 17 (E7), 1-9.

Tokat, M.A., Okumuş, H., Dennis, C.L., 2010. Translation and psychometric assessment of the Breast-feeding Self-Efficacy Scale-Short Form among pregnant and postnatal women in Turkey. Midwifery 26 (1), 101-108.

Torres, M.M., Torres, R.R.D., Rodríguez, A.M.P., Dennis, C.L., 2003. Translation and validation of the breastfeeding self-efficacy scale into Spanish: data from a Puerto Rican population. Journal of Human Lactation 19 (1), 35-42.

Van Rossem, L., Oenema, A., Steegers, E.A., et al., 2009. Are starting and continuing breastfeeding related to educational background? The generation R study. Pediatrics 123 (6), e1017-e1027.

Wheeler, B.J., Dennis, C.L., 2013. Psychometric testing of the modified breastfeeding self-efficacy scale (short form) among mothers of Ill or preterm infants. Journal of Obstetric, Gynecologic, \& Neonatal Nursing 42 (1), 70-80.

World Health Organization, \& UNICEF. (2003). Global strategy for infant and young child feeding. World Health Organization.

Wutke, K., Dennis, C.L., 2007. The reliability and validity of the Polish version of the breastfeeding self-efficacy scale-short form: translation and psychometric assessment. International Journal of Nursing Studies 44 (8), 1439-1446.

Zubaran, C., Foresti, K., Schumacher, M., et al., 2010. The Portuguese version of the breastfeeding self-efficacy scale-Short form. Journal of Human Lactation 26 (3), 297-303. 